\title{
Effects of Peptide Backbone Amide-to-Ester Bond Substitution on the Cleavage Frequency in Electron Capture Dissociation and Collision-Activated Dissociation
}

\author{
Frank Kjeldsen, ${ }^{1}$ Roman A. Zubarev ${ }^{2,3}$ \\ ${ }^{1}$ Department for Biochemistry and Molecular Biology, University of Southern Denmark, Odense, Denmark \\ ${ }^{2}$ Department of Molecular Biochemistry and Biophysics, Karolinska Institutet, Stockholm, Sweden \\ ${ }^{3}$ Science for Life Laboratory, Stockholm, Sweden
}

\begin{abstract}
Probing the mechanism of electron capture dissociation on variously modified model peptide polycations has resulted in discovering many ways to prevent or reduce $\mathrm{N}-\mathrm{C}_{\alpha}$ bond fragmentation. Here we report on a rare finding of how to increase the backbone bond dissociation rate. In a number of model peptides, amide-to-ester backbone bond substitution increased the frequency of $\mathrm{O}-\mathrm{C}_{\alpha}$ bond cleavage (an analogue of $\mathrm{N}-\mathrm{C}_{\alpha}$ bonds in normal peptides) by several times, at the expense of reduced frequency of cleavages of the neighboring $\mathrm{N}-\mathrm{C}_{\alpha}$ bonds. In contrast, the ester linkage was only marginally broken in collisional dissociation. These results further highlight the complementarity of the reaction mechanisms in electron capture dissociation (ECD) and collision-activated dissociation (CAD). It is proposed that the effects of amide-to-ester bond substitution on fragmentation are mainly due to the differences in product ion stability (ECD, CAD) as well as proton affinity (CAD). This proposal is substantiated by calculations using density functional theory. The implications of these results in relation to the current understanding of the mechanisms of electron capture dissociation and electron transfer dissociation are discussed.
\end{abstract}

Key words: Electron capture dissociation, Depsipeptides, Cleavage frequencies, Fragmentation mechanism of electron capture dissocation

\section{Introduction}

mino acid sequencing of peptide and protein ions by
tandem mass spectrometry (MS/MS) is an essential part
of most proteomics studies [1, 2]. In the MS/MS events,
fragmentation of polypeptide ions is typically performed by
collision-activated dissociation (CAD) to provide sequence-
specific product ions that are used for protein identification
via database searching. An alternative to CAD fragmentation
is electron capture dissociation (ECD) [3] and its more
recent analogue electron transfer dissociation (ETD) [4].
These latter two techniques are based on the reaction of low-

Correspondence to: Frank Kjeldsen; e-mail: frankk@bmb.sdu.dk energy electrons with multiply charged cations, and they have become very popular in proteomics. Much of the popularity stems from the fact that these electron-based fragmentation techniques can cleave backbone bonds in modified polypeptide cations with no or minimal loss of labile modifications [5-7]. Both ECD and ETD cleave preferentially the $\mathrm{N}-\mathrm{C}_{\alpha}$ backbone bond in peptide polycations producing $\mathrm{c}^{\prime}$ - and $\mathrm{z}$-type fragment ions (notation from reference [8]; prime sign denotes hydrogen atom addition compared with homolytic cleavage), while CAD [9] and other slow-heating techniques cleave predominantly the $\mathrm{C}-\mathrm{N}$ bond, producing $\mathrm{b}$ - and $\mathrm{y}^{\prime}$-type ions. Both the type of ECD/ETD bond cleaved as well as the preferred cleavage sites in the peptide sequence are complementary to CAD [10]. This complementarity allows for more reliable protein 
identification when both CAD and ECD/ETD techniques are applied to the same polypeptide [11]. Analytical benefits of ECD/ETD in proteome research have been demonstrated in a number of studies $[12,13]$.

Due to their relative novelty and importance in mass spectrometry and proteomics, ETD, and in particular ECD have been a subject of a number of experimental and theoretical studies. Many studies have focused on understanding the underlying mechanisms of inter-residue bond cleavage. These mechanisms involve at least two crucial moments. First, what factors influence the formation of the unstable amino ketyl radical that is believed to be an intermediate for the $\mathrm{N}-\mathrm{C}_{\alpha}$ bond cleavage [14]? The formation of this intermediate is lively debated, especially the factors concerning the location of the initial electron attachment to peptide cations [15-20]. The second critical step encompasses the fragmentation reactions occurring after formation of the amino ketyl radical, such as the losses of atomic hydrogen, side chains [21] or $\mathrm{N}-\mathrm{C}_{\alpha}$ bond cleavage via primary of secondary $[22,23]$ radical reactions. These fragmentation pathways are believed to be largely determined by the energy levels of the formed products as well as their transition states $[15,16]$.

The first suggested ECD mechanism proposed electron capture by a positively charged ammonium or guanidinium functional group solvated by a backbone carbonyl resulting in a hypervalent site and hydrogen atom addition to that carbonyl. The resulting amino ketyl radical is unstable and undergoes radical-driven $\alpha$-cleavage of the $\mathrm{N}-\mathrm{C}_{\alpha}$ bond, which produces c- and z-type fragments [3]. This dissociation mechanism is often referred to as the Cornell mechanism. In its later variant, the hydrogen atom is released by the hypervalent site and then recaptured by a more distant site with high hydrogen affinity (HA), such as a backbone carbonyl or S-S bond. This model is often referred as a "hot hydrogen atom" mechanism [24]. Since the cross-section for direct capture of free hydrogen atoms by gas-phase peptides is quite low [25], it appears that mainly hydrogen atoms from neutralized protonated groups solvated to carbonyls can be effectively transferred to carbonyl oxygens [23]. An alternative mechanism was put forward independently by Turecek and Simons and coworkers [26-28]. This mechanism suggests electron capture into the amide OCN $\pi^{*}$ orbital. The anionic site formed by this capture requires stabilization by the electrostatic field of a nearby solvated proton. It has been argued that the electron capture to the amide $\pi^{*}$ orbital increases the basicity of the amide carbonyl oxygen to $1100-1400 \mathrm{~kJ} / \mathrm{mol}$, and thus the formed radical anion is able to abstract a proton from not only distant protonated, but even neutral, sites in the molecule, perhaps via a conformational change in the molecule [28]. This series of events is usually referred to as the Utah-Washington (UW) model. The Uppsala group have pointed out that the large barrier for proton rearrangement and long (nanoseconds) time scale of conformational changes might make distant proton migration kinetically unfavorable compared with the much faster (picoseconds) electron transfer to the protonation site [20]. However, if the carbonyl is already engaged in intramolecular neutral hydrogen bonding, hydrogen atom transfer from the H-bond donor to the carbonyl over $\sim 1 \AA$ distance can kinetically dominate (a hydrogen atom at room temperature covers this distance in ca. $40 \mathrm{fs}$ ). Such an H-bond based "Uppsala model" could quantitatively reproduce ECD fragment abundances for four related polypeptide molecules [20]. Currently, there seems to be a consensus that different mechanisms capture different aspects of such a vastly complex phenomenon as electron capture (transfer) dissociation.

Probing the ECD mechanism on variously modified model peptide polycations has resulted in discovering many ways to prevent or reduce $\mathrm{N}-\mathrm{C}_{\alpha}$ bond fragmentation in ECD. O'Connor and co-workers have studied the role of radicals in ECD by chemically adding to peptides a variety of tags which act as free radical traps [29, 30]. In those studies, the addition of one or two radical traps to doubly charged peptides significantly reduced or blocked the ECD backbone cleavage channels. Turecek and co-workers prevented ECD backbone cleavages by the addition of a stable electron and hydrogen atom trap to peptide ions [31]. More recently, Beauchamp and co-workers inserted tags with variable electron affinities (EA; -1.15 to $+1.65 \mathrm{eV}$ ) into a peptide sequence and analyzed the model peptides by ECD and ETD [32]. Two main conclusions were drawn. First, the EA of the tags had no influence on the electron capture cross-section of peptide ions. Second, ECD fragmentation was inhibited with substituent tags having EA above $0.50 \mathrm{eV}$. It was concluded that these results support the idea that initial electron capture happens preferentially at one of the positive sites with subsequent through-space or through-bond electron transfer to the tags of high electron affinity in competition with transfer to the amide $\pi^{*}$ orbital.

These and other studies have highlighted the importance in ECD of functional groups with either high electron or proton affinities. However, much of the information gained by the tagging approach is limited to the chemistries of the tags. Ideally, the change in the peptide sequence and structure should be small, so that the induced changes can easily be attributed to one or a few changed parameters (an example is L/D-conversion of the amino acid chirality). In the present study, we pursued this idea and designed a peptide model system that is close to natural peptides. This approach is to selectively substitute a peptide amide bond (-CO-NH- group) with an ester bond (-CO-O- group). Natural peptides with such bonds exist; they are called depsipeptides [33]. The ester bond adopts similar bond angles and lengths, prefer similar $\Phi$ and $\Psi$ dihedral angles, and also strongly prefer trans-ester conformation like secondary amides [34]. But, compared with the amide, the carbonyl in the ester group is a poorer hydrogen acceptor due to its much lower proton affinity [35]. Differences between amide and ester bonds are also found in this study for the vertical electron affinity (VEA), gas-phase basicity of 
the radical anions, and bond dissociation energies (BDE) of the radical intermediates of the ester and amide motif. Therefore, ester bond substitution can induce a small but significant change in the peptide gas-phase structure and property.

ECD of peptides with ester bonds has previously been investigated [36, 37]. Marshall and co-workers have found that backbone cleavages in cyclic all-ester depsipeptides mainly occur in the $\mathrm{O}-\mathrm{C}_{\alpha}$ bond [36], while Guan investigated fragmentation of the ester bond in a side chain [37]. Backbone ester bond dissociation gives $z$-type fragment ions similar to their all-amide counterparts, while the complementary N-terminal fragments correspond to $\mathrm{N}$ terminal truncated peptides (R-COOH type) as shown in Scheme 1. Note that in CAD of all-amide peptide ions, $y^{\prime}$ ions are truncated C-terminal peptides, while $b$-ions are dehydrated peptides. In CAD and other processes leading to fragmentation (e.g., MALDI), $b+\mathrm{H}_{2} \mathrm{O}$ fragments sometimes appear for all-amide peptides having a carboxylic Cterminus $[38,39]$. Technically, these sporadically emerging species are similar in structure to the N-terminal ECD fragments of the ester bond, but the latter ions are present in ECD mass spectra systematically and, as will be shown, often with high intensity. Therefore, we found it more logical to denote these species $\mathrm{c}^{\# \prime}$ ions (in [36], they are called $c$ ions).

In this work, we compare ECD cleavage frequencies of amide bonds before and after substitution by an ester bond and demonstrate that, as a rule, ester bond substitution increases the frequency of ECD cleavages. The possible origin of this phenomenon is investigated using density functional theory calculations on model systems.

\section{Experimental}

\section{Synthesis}

Peptides were synthesized using a research micro-scale ResPep peptide synthesizer (Intavis AG, Gladbach, Germany) configured for the $\mathrm{N}-\alpha$-Fmoc synthesis protocol [40] and optimized for $5 \mu \mathrm{mol}$ yields of peptides. For the synthesis of C-terminal carboxylic peptides, the preloaded TGA resin TentaGel (Novabiochem, Darmstadt, Germany, capacity $0.1-0.3 \mathrm{mmol} / \mathrm{g}$ ) was used. Activation of the $\alpha$ carboxy group for coupling of the amino acids was done with 0.9 M PyBOP (benzotriazole-1-yl-oxy-tris-pyrrolidininophosphonuim hexafluorophosphate) (Calbiochem-Novabiochem, Läufelfingen, Switzerland). Coupling was catalyzed by $N$-methylmorpholine (NMM) (Fluka, Buchs SG,
Switzerland) in $\mathrm{N}, \mathrm{N}$-dimethylformamide (DMF) (Fluka). Standard coupling time was 15 minutes with double couplings after the ninth cycle, each lasting 20 minutes. Fmoc deprotection between each coupling cycle was done with $20 \%$ piperidine (Riedel-de Haën, Seelze, Germany) in DMF (technical grade, Fluka) for $2 \times 5 \mathrm{~min}$ and for $2 \times 10 \mathrm{~min}$ after cycle nine. Between each step, the resin was washed with DMF (technical grade). After completion of the synthesis, the resin was washed with ethanol and dichloromethane (Fluka) and dried over a dry nitrogen flow for $5 \mathrm{~min}$. Cleavage of the peptide from the resin and side chain deprotection was performed at room temperature in a mixture of trifluoroacetic acid (Fluka), triisopropylsilane (Fluka) and MilliQ-water (Millipore) MA, USA $(95: 2.5: 2.5 ; \mathrm{vol} / \mathrm{vol} / \mathrm{vol})$ for $2 \mathrm{~h}$. Peptides and depsipeptides were precipitated with cold $\left(-21^{\circ} \mathrm{C}\right)$ tert-butyl-methylether (Fluka) and centrifuged for $2 \mathrm{~min}$, after which the supernatant was removed. The peptide precipitate was finally reconstituted in $70 \%$ acetonitrile and speed-vacuumed to dryness.

For the synthesis of depsipeptides, NMM was substituted with the more sterically hindered tertiary amine DBU $(1,8-$ diazabicyclo[5.4.0]undec-7-en) (1\% in dry DMF) to prevent hydrolysis of the ester-bond. The synthesis strategy was based on the coupling of an ester-bound di-depsipeptide to the extending peptide chain (Scheme 2).

The di-depsipeptide was presynthesized manually by activating the Fmoc-amino acid $(0.13 \mathrm{mmol})$ with PyBoP $(0.125 \mathrm{mmol})$ for $10 \mathrm{~min}$ at RT in NMP $(50 \mu \mathrm{L})$ and NMM $(25 \mu \mathrm{L}) ; 0.13 \mathrm{mmol}$ of the $\alpha$-hydroxyl equivalent to the amino acid of Gly and Val was added and then stirred for 60 min at RT (Scheme 3).

Typically, the peptide yields ranged from $60 \%$ to $80 \%$, whereas the depsipeptide synthesis yield was between $1 \%$ and $10 \%$.

\section{Mass Spectrometry}

A 7 T ESI LTQ-FT mass spectrometer (Thermo Electron, Bremen, Germany) equipped with an indirectly heated dispenser cathode as an electron source was used for ECD experiments. All spectra were obtained in broadband detection mode with a resolution of 100,000 at $\mathrm{m} / \mathrm{z}$ $400 \mathrm{Da}$. Molecular ions were isolated in the linear ion trap and fragmented by collisions with $\mathrm{He}(\mathrm{g})$ or transferred to the Penning (ICR) trap for ECD experiments. An electron current of $30 \mu \mathrm{A}$ was used in all experiments; the irradiation time was $70 \mathrm{~ms}$ with electron energy below $1 \mathrm{eV}$. In each

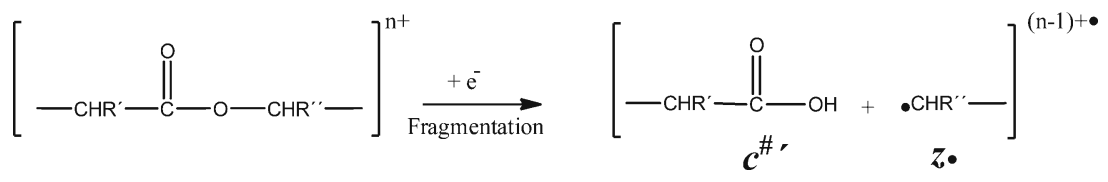




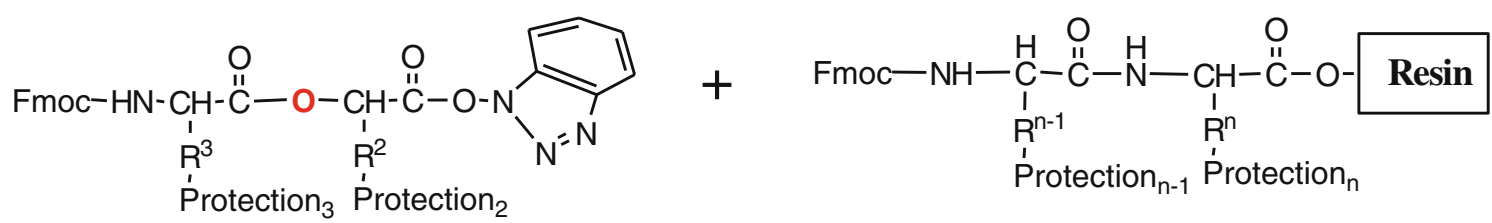

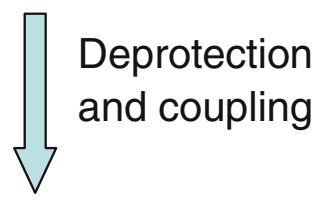

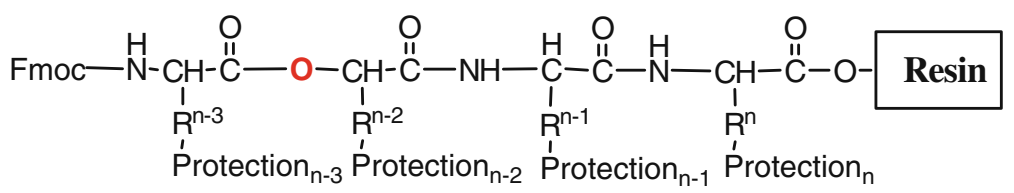

Scheme 2. Solid-phase coupling of a di-depsipeptide derivative to the extending peptide chain

experiment, a total of at least five acquisitions containing 10 individual MS/MS spectra each were averaged.

\section{Calculations}

Standard density functional theory calculations were performed with the Gaussian 03 suite of programs [41]. Optimized geometries of the model species relevant to this study were obtained using the B3LYP level of theory and a $6-31+G(d, p)$ basis set. For higher accuracy, single-point energies of optimized structures were calculated with the same level of theory using a $6-311++G$ (2df,2p) basis set after adding both polarization and diffuse functions for heavy atoms and hydrogens. The harmonic vibrational frequencies of stationary points on the potential energy surface (PES) were calculated at the same level of theory as used for their optimization in order to identify local minima (all real frequencies).
Zero-point energies (ZPE) were computed by adjusting harmonic frequencies by an appropriate correction factor of 0.9804 for the B3LYP level of theory. The zero-pointcorrected energies were then combined with the total energies resulting from the geometry optimizations. VEA values were calculated using a modified version of Gaussian on the Opteron Arches Clusters at the University of Utah using MP2 and a 6-31 G(d,p) basis set. This version of Gaussian is particularly suited for calculations of anions that are not electronically stable. This was achieved by using a nuclear charge stabilization method [42, 43]. Briefly, in order to obtain an estimate of the (negative) electron binding energy of the species in question, the nuclear charge of the nuclei involved in the electron attachment was altered by small amounts of $\delta q$. Then, a series of calculations was performed on the neutral and anion energy separation as a function of $\delta \mathrm{q}$. Finally, the energy differences (neutral-anion) were

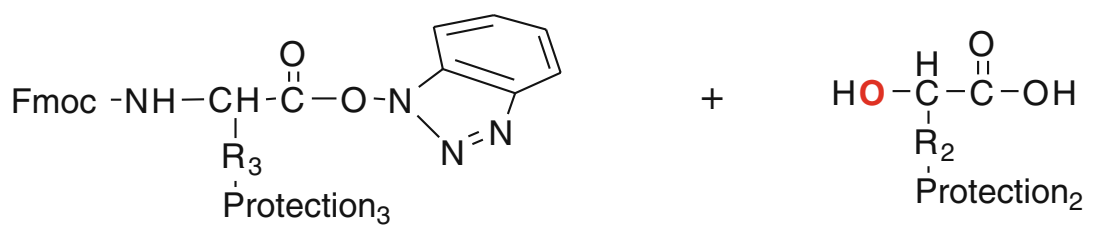

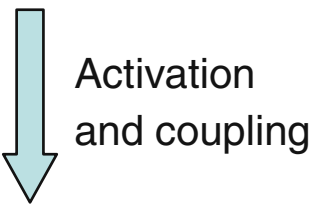<smiles>O=C(On1nnc2ccccc21)C(COC(=O)C(NCC(F)F)PPOCO)P[PH]</smiles>

Scheme 3. Synthesis of the di-depsipeptide derivative through activation of the Fmoc-amino acid and subsequent reaction with the a-hydroxyl equivalent to the amino acids of Gly and Val 
plotted against $\delta q$. For this plot, we used only $\delta q$ values large enough to satisfy [E(neutral)-E(anion) > 0]. In order to obtain an estimate of the EA, the trend was extrapolated to $\delta \mathrm{q}=0$.

\section{Results}

Several synthesized depsipeptides with the $\alpha$-hydroxyl equivalents of Gly (2-hydroxyacetic acid) or Val (2-hydroxy-3methylbutanoic acid) (the bond substitution resulted in formation of the sequences -Aaa-COO-Gly/Val-) were fragmented by CAD and ECD. For comparison, regular peptides with the same sequences were fragmented by the same techniques.

\section{Substance P and Its Depsipeptide Analogue}

Figure 1 shows the ECD mass spectra of Substance P 2+ ions with all-amide linkages (top) and with an ester-linkage between the $\mathrm{Phe}^{8}$ and $\mathrm{Gly}^{9}$ residues (bottom). The product mass spectra are dominated by $c^{\prime}$ type ion series from $c^{\prime}{ }_{4}$ to $\mathrm{c}^{\prime}{ }_{10}$. A distinct feature of the depsipeptide ECD mass spectrum is a more than twice enhanced cleavage frequency of the ester-linkage $\left(\mathrm{c}^{\#_{1}} 8\right)$ compared with that in the native sequence. At the same time, adjacent cleavages next to the ester-linkage ( $\mathrm{c}_{7}{ }_{7}$ and $\mathrm{c}_{9}{ }_{9}$ ) were reduced by approximately $60 \%$ each.

In ECD spectra of 3+ ions (Figure 2), the fragment ion intensities were normalized to the $\mathrm{c}_{5}{ }_{5}$ abundances. The ester bond substitution has qualitatively the same effect on ECD of $3+$ ions as $2+$ species: the $\mathrm{O}-\mathrm{C}_{\alpha}$ bond cleavage is 3.5 times more frequent than $\mathrm{N}-\mathrm{C}_{\alpha}$ cleavage. The frequencies of neighboring cleavages were reduced by $45 \%-50 \%$.

CAD fragmentation of Substance $\mathrm{P}$ and its depsipeptide analogue reveals a complementary to ECD behavior: 2+ ions gave almost complete absence of $\mathrm{C}-\mathrm{O}$ cleavages in the depsipeptide analogue (gray bar in Figure 3). Compared with the $2+$ all-amide Substance $P$, the cleavage frequency of the ester linkage is reduced by a factor of 22 ! The above observations are in good agreement with the results reported by Guan on the acylated peptide Ghrelin [37]. In that study, the acylated fatty acid peptide also showed abundant loss of octanoic acid (ester bond cleavage) from the precursor ions in ECD as well as a limited cleavage of the ester bond in CAD.

\section{ECD of LYYVYLVR and LVY-COO-VYLVR}

In ECD of the tryptic model peptide LVYVYLVR and its depsipeptide analogue LVY-COO-VYLVR (Figure 4), two

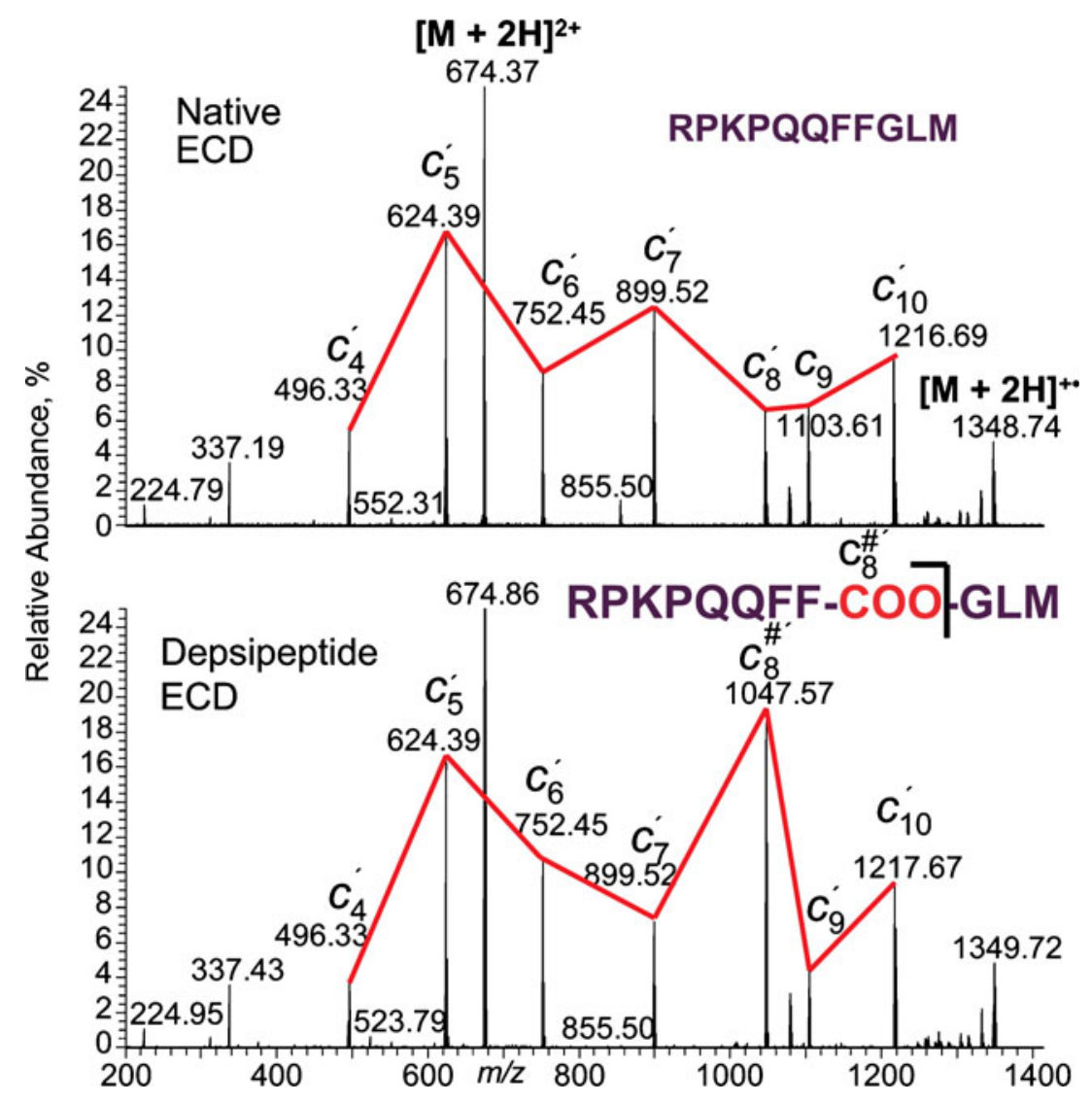

Figure 1. ECD mass spectra of dications of Substance $P$ (top) and the depsipeptide analogue of Substance $P$ (bottom) with an ester linkage between $\mathrm{Phe}^{8}$-COO-Gly ${ }^{9}$. The cleavage frequency of the ester linkage is 2.7 times more abundant than the corresponding amide bond in Substance $\mathrm{P}$ 


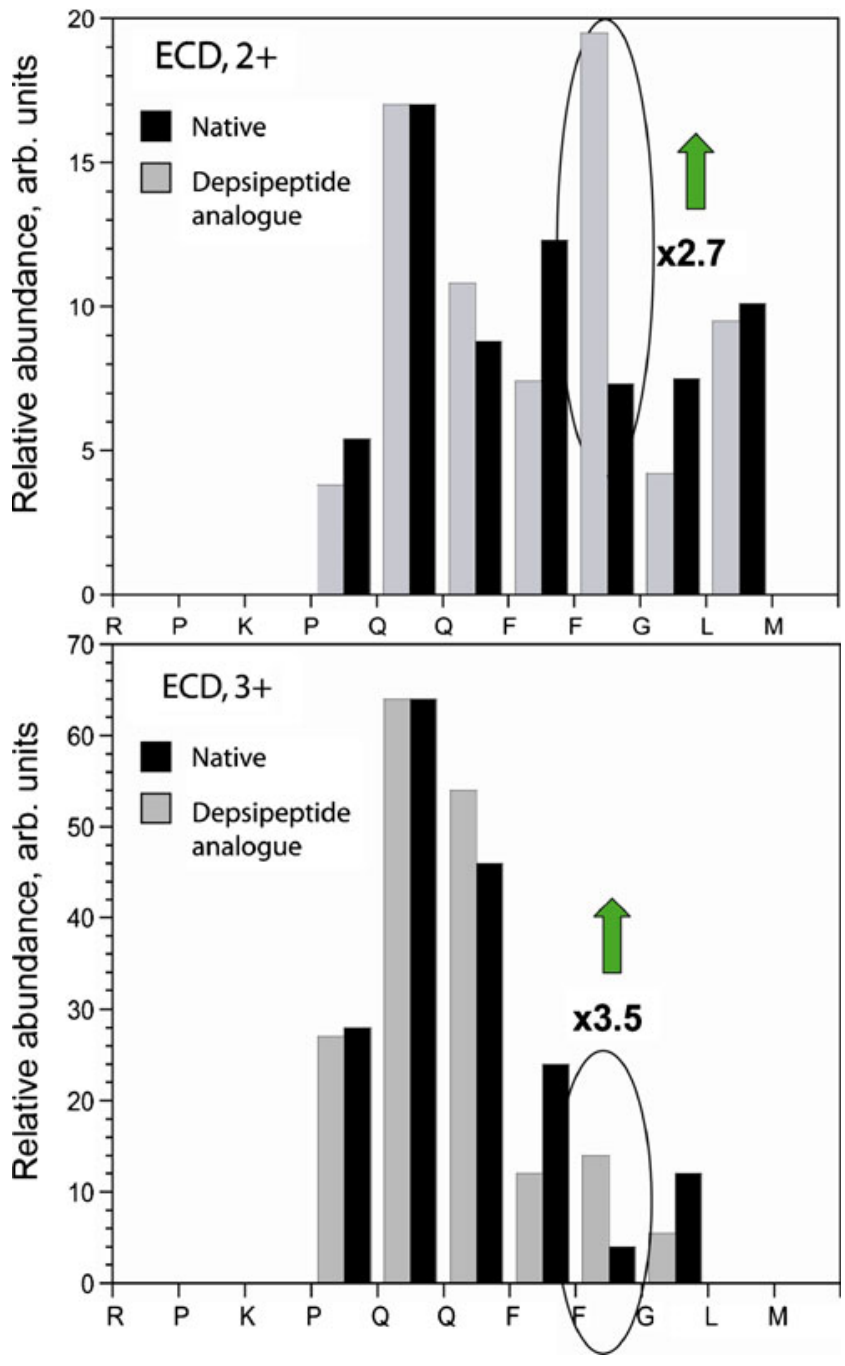

Figure 2. Bar diagram of the relative product ion abundances in ECD of native Substance $P$ (all-amide) and its depsipeptide analogue in precursor charge state $2+$ (top) and $3+$ (bottom)

distinct differences are present. While the all-amide peptide produces predominantly $\mathrm{z}$-type fragment ions $\left(\mathrm{z}_{3}{ }_{3}, \mathrm{z}^{\cdot}{ }_{4}, \mathrm{z}_{5}, \mathrm{z}_{6}\right.$, and $\left.z_{7}{ }_{7}\right)$ together with two $b$ ions $\left(b_{6}\right.$ and $\left.b_{7}\right)$ and one $c$ ion $\left(c^{\prime}\right)$, the depsipeptide variant gave only two backbone bond fragments $\mathrm{z} \cdot{ }_{3}$ and $\mathrm{z} \cdot{ }_{5}$, with $\mathrm{z} \cdot{ }_{5}$ arising from the breakage of the ester bond. In addition, the depsipeptide did not yield any small-loss ions, which also stands in sharp contrast to the all-amide peptide. The abundance of the fragment arising from the ester bond cleavage was only marginally increased compared with that of the corresponding amide bond cleavage.

ECD of KTVGVGVGVGTK and KTVGVGVGVG-COO-VGTK

Similar to the previous examples, ECD spectra of KTVGVGVGVGTK and its depsipeptide analogue (KTVGVGVGVG-COO-VGTK) display significantly dif-

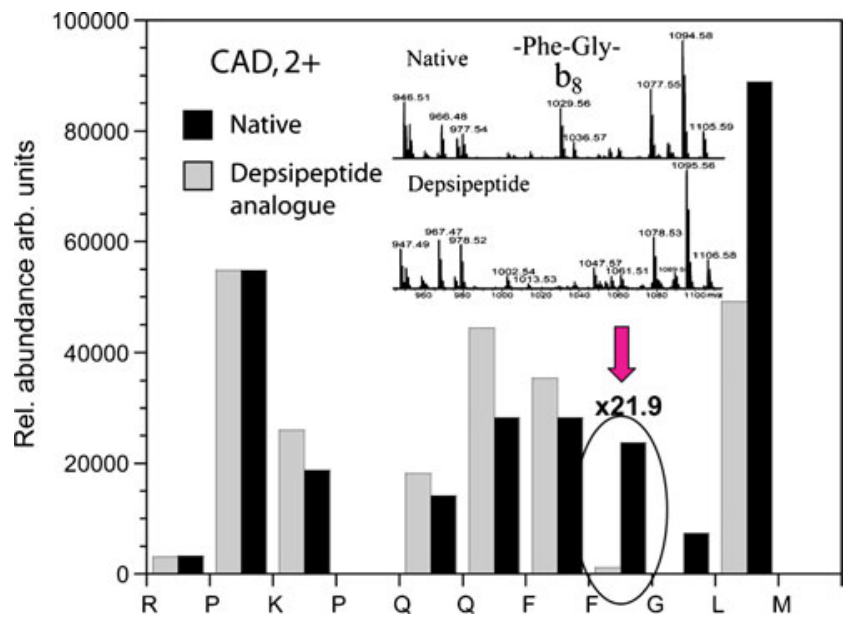

Figure 3. Bar diagram of the product ion abundances in CAD from 2+ precursors of native Substance $P$ (all-amide) and its depsipeptide analogue ( $\left.\mathrm{Phe}^{8}-\mathrm{COO}-\mathrm{Gly}^{9}\right)$. Inset shows the marginal cleavage of the ester bond in CAD $\left(b_{8}\right)$ compared with the fifth most abundant backbone cleavage for the all-amide Substance $P$

ferent mass spectra (Figure 5). While the ester bond cleavage frequency represented by the $\mathrm{c}_{8}^{\#_{1}}$ fragment ions was increased compared with the all-amide peptide, there is a significant decrease in the cleavage frequencies of the amide bonds neighboring the ester backbone bond. This is clearly demonstrated by the absence of $\mathrm{c}_{9}{ }^{\prime}$ and a $50 \%$ reduction in the $c_{7}{ }_{7}^{\prime}$ ion abundance. In fact, all c-type ions except $\mathrm{c}^{\#_{1}}{ }_{8}$ are reduced in abundance. In addition, the stability of the reduced species of the depsipeptide is greatly reduced in respect to that of the allamide analogue and a complete absence of small losses is observed. Perhaps the most striking difference of the two spectra is the almost complete absence of C-terminal z-type ions in the depsipeptide ECD spectrum. In the ECD spectrum of the all-amide analogue, this ion type represents about $35 \%$ of the product ion population.

Summarizing the observations for all three peptidedepsipeptide pairs, the abundance of the ester bond cleavage relative to other backbone cleavages in ECD is significantly increased, while those of other peptide bonds are somewhat reduced, with the most significant reduction observed at the sites next to the ester link.

To rationalize these observations, we performed thermodynamic calculations of two model systems representing the amide and the ester linkages. Assuming that the ECD/ETD mechanism is mostly driven by thermodynamics, we calculated different parameters of the two model systems and considered those with the largest energy differences, searching among them for the explanation of the observed effects.

\section{Density Functional Theory Calculations of N-Methyl Acetamide and Methybl Acetate}

Figure 6 shows the comparison of thermodynamic energies for the structurally optimized reaction species of $\mathrm{N}$-methyl 


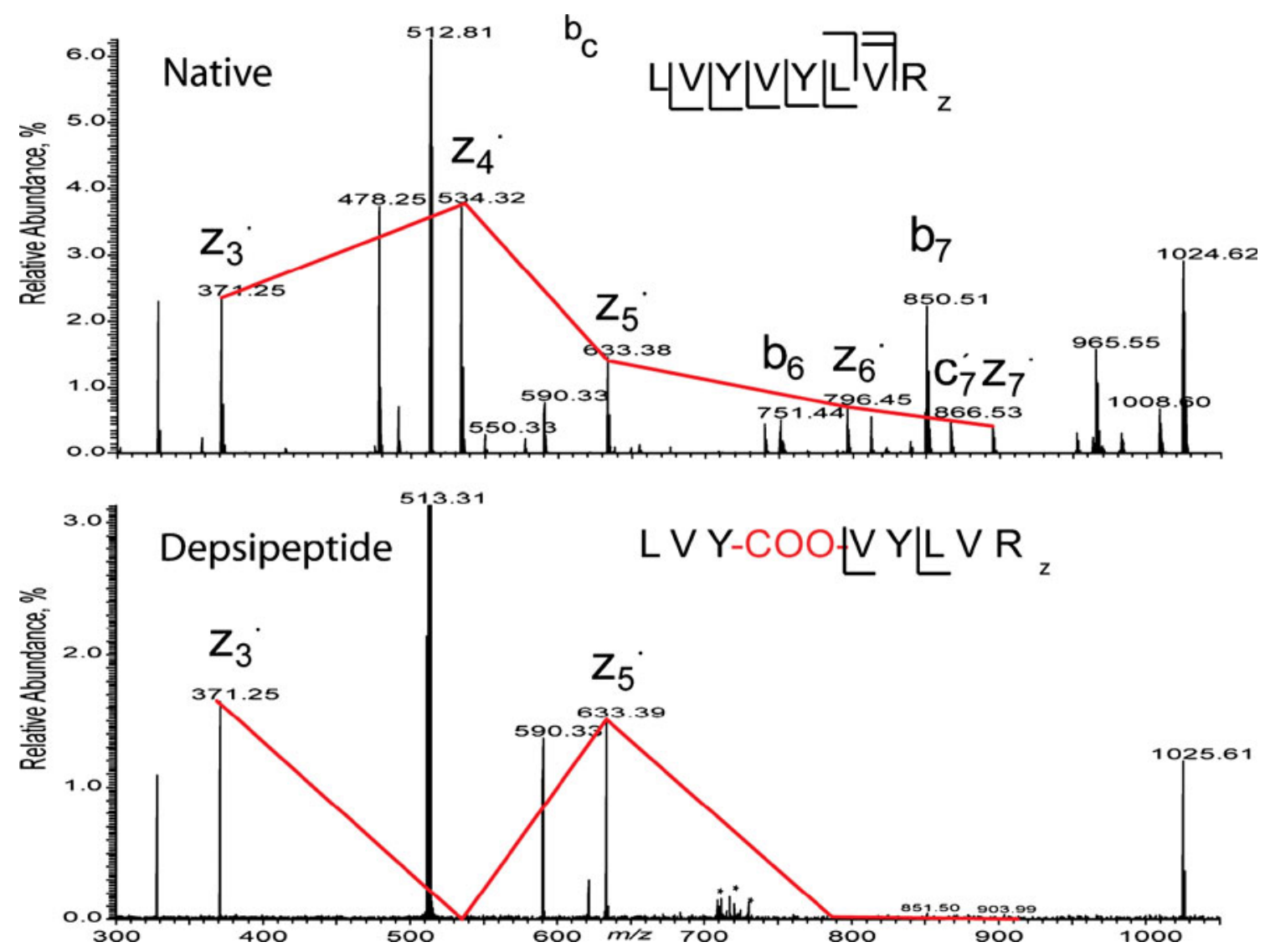

Figure 4. ECD mass spectra of 2+ precursor ions of the peptide LVYVYLVR and its depsipeptide analogue LVY-COO-VYLVR

acetamide and methyl acetate. For proton affinity (PA) evaluation, local minima structures with different cis/trans and syn/anti configurations were calculated. In agreement with Turecek and Yao, the protonated trans-anti configuration was found to be the lowest in energy [44]. In good agreement with their study, we determined the PA for the carbonyl oxygen of $\mathrm{N}$-methyl acetamide (2a) to be $895 \mathrm{~kJ} / \mathrm{mol}$. The PA of the carbonyl oxygen in methyl acetate (2b) was found substantially lower at $826 \mathrm{~kJ} / \mathrm{mol}$. In both models, the carbonyl oxygens are the most basic sites, which points to these sites being favored for involvement in the solvation of charges in the gas-phase. From the PA alone, it is expected that the participation in charge solvation of the ester carbonyl oxygen is greatly reduced compared with that of the amide carbonyl oxygen. This is also consistent with the calculated strengths of neutral hydrogen bonding between one molecule of N-methyl acetamide and one molecule of methyl acetate $(7 \mathrm{~kJ} / \mathrm{mol})$ versus that of two molecules of N-methyl acetamide $(12 \mathrm{~kJ} / \mathrm{mol})$ determined at the B3LYP/3-21 G//6-311+G(2 d,p) level of theory and basis set (data not shown).

Vertical electron affinities (VEA) for neutral N-methyl acetamide and methyl acetate were found endothermic with $-419 \mathrm{~kJ} / \mathrm{mol}$ (3a) and $-365 \mathrm{~kJ} / \mathrm{mol}$ (3b), respectively. Literature EA for N-methyl acetamide is not available. The next best comparison is made to formamide, which is found endothermic at $-199 \mathrm{~kJ} / \mathrm{mol}$ [45]. The literature EA value of methyl acetate is $-145 \mathrm{~kJ} / \mathrm{mol}$ [46]. Since experimentally obtained EA values are notoriously difficult to reproduce using DFT calculations [47], it is not surprising that these calculations underestimated the VEA values. However, the relative difference in VEA of $54 \mathrm{~kJ} / \mathrm{mol}$ between $\mathrm{N}$-methyl acetamide and methyl acetate is considered to be within a realistic magnitude given the strikingly identical EA difference $(54 \mathrm{~kJ} / \mathrm{mol})$ found between formamide and methyl acetate.

The addition of a hydrogen atom to $\mathbf{1 a}$ and $\mathbf{1 b}$ releases $62 \mathrm{~kJ} / \mathrm{mol}$ and $34 \mathrm{~kJ} / \mathrm{mol}$, respectively [hydrogen atom affinity (HA) of the amide and ester groups, respectively]. Interestingly, the bond dissociation energy (BDE) is significantly different for $\mathbf{4 a}$ and $\mathbf{4 b}$. The amino ketyl radical (4a) is $2 \mathrm{~kJ} / \mathrm{mol}$ endothermic in respect to $\mathrm{N}-\mathrm{C}_{\alpha}$ bond cleavage. In contrast, the ketyl radical (4b) is exothermic by $96 \mathrm{~kJ} / \mathrm{mol}$ for cleavage of the $\mathrm{O}-\mathrm{C}_{\alpha}$ bond. As the radical methyl is the same leaving group from both $\mathbf{4 a}$ and $\mathbf{4 b}$, the significant weakening of the ester bond compared with the amide bond can be related to the stability of the formed carboxylic acid versus the enol amine. Transition state geometries for conversion of $\mathbf{4 a}$ to $\mathbf{5 a}$ and $\mathbf{4 b}$ to $\mathbf{5 b}$ were not investigated. However, Turecek and co-workers determined the energy barrier for $\mathrm{N}-\mathrm{C}_{\alpha}$ bond cleavage 
for the amino ketyl radical of N-methyl acetamide to be $84 \mathrm{~kJ} / \mathrm{mol}$ [44]. In another study, Turecek and coworkers found a qualitative relationship between the decrease in BDE and the reduction in the activation energy for $\mathrm{N}-\mathrm{C}_{\alpha}$ bond cleavage [48]. The reduction in BDE was explained by the increased stability of the products. In the case of the ester bond in depsipeptides, the formed carboxylic acid is much more stable than the enol amine. Hence, it can be speculated that the energy barrier for $\mathrm{O}-\mathrm{C}_{\alpha}$ bond cleavage in the ketyl radical (4b) is also substantially lower than the $\mathrm{N}-\mathrm{C}_{\alpha}$ bond cleavage in the amino ketyl radical (4a). This raises the possibility that $\mathrm{O}-\mathrm{C}_{\alpha}$ bond cleavage can occur exothermically without crossing any energy barrier.

Concerning the electron affinities (VEAs) of the two model systems, VEA of the ester bond is larger by $\triangle \mathrm{VEA}=54 \mathrm{~kJ} / \mathrm{mol}$. However, the basicity of the radical anions formed upon electron capture is smaller: the basicity of the N-methyl acetamide "super-bases" 3a are $1559 \mathrm{~kJ} / \mathrm{mol}$ and for methyl acetate $3 \mathbf{b}$ it is $1484 \mathrm{~kJ} / \mathrm{mol}$. In fact, this difference between the basicities of the two radical structures is the largest in the calculations $(75 \mathrm{~kJ} / \mathrm{mol})$. As for the ECD fragment stabilities, they are $71 \mathrm{~kJ} / \mathrm{mol}$ in favor of methyl acetate.

\section{Discussion}

\section{ECD Results are Best Explained by Different Product Stabilities}

Different ECD mechanisms emphasize the importance of different parameters, and thus account for the observed effects with different degrees of success. The Cornell mechanism requires efficient solvation of the protonated groups on backbone carbonyls and thus the reduction in basicity by $69 \mathrm{~kJ} / \mathrm{mol}$ of the ester bond poses a problem in explaining its enhanced cleavage (charge solvation on the $\mathrm{O}$ - functionality in the ester bond is unlikely because of the much lower proton affinity of that group compared with that of the carbonyl oxygen). But, on the other hand, electroncharge recombination most frequently occurs in ECD at the sites with the highest recombination energy (which are also the least basic sites) [49] and, thus, the lesser basicity of the ester bond can result in its more frequent recombination when it solvates a protonated group. The hot hydrogen atom model focuses on hydrogen atom affinity, which is higher for amide, which again poses a difficulty. On the other hand, $\mathrm{H}$ - capture by the $-\mathrm{O}$ - group will probably immediately cleave that bond, although no evidence exists that such capture can occur at a high rate. The Utah-Washington (UW) model, where electron affinity of the carbonyl plays a decisive role, benefits from the higher VEA $(54 \mathrm{~kJ} / \mathrm{mol})$ of the backbone ester bond. Thus, the anion formed by the ester carbonyl requires less Coulomb stabilization than the OCN $\pi^{*}$ orbital. However, Beauchamp and co-workers [32] showed that electron capture and cleavage of amide bonds were only affected when substituent tags in the peptide had
EA greater than ca. $50 \mathrm{~kJ} / \mathrm{mol}$. Therefore, it is unclear whether the difference in VEA is enough to shift the balance decisively in favor of electron capture at the ester bonds. Also, the more basic amide radical anion should more easily attract protons, which in the UW model should benefit backbone fragmentation of amides over esters. The Uppsala model stresses the importance of neutral hydrogen bonding, but ester's carbonyls are less efficient H-bond acceptors than amide's carbonyls, while the -O- group obviously cannot be a H-bond donor.

Recently, the Tsybin group has demonstrated correlation between ECD cleavage frequency and stability of formed radical products [50]. Previously, product stability has often been suggested in theoretical literature as one of the major driving forces behind the cleavage of a particular bond (e.g., [48]). In that respect, ester bonds have a clear advantage over the amide bonds. As mentioned, the reduction in $\mathrm{BDE}$ for the ketyl radical can be explained by the formation of the more stable carboxylic acid (5b) compared with the enol imine (5a) formed from the amino ketyl radical. Even if sufficient excess energy exists in the latter species to cross the barrier and rearrange the enol imine to the more stable amide form, the difference in BDE would still favor the ester bond cleavage by $18 \mathrm{~kJ} / \mathrm{mol}$ (data not shown).

Besides the increased cleavage frequency, another effect of the amide/ester substitution on ECD mass spectra is the shift in the charge state distribution of fragments, which in the case of doubly charged precursors means shifting the balance between N- and C-terminal fragments. For instance, in ECD of KTVGVGVGVGTK and its depsipeptide analogue KTVGVGVG-COO-VGTK, the former provides a series of z-fragments, and the latter demonstrates their complete absence (Figure 5). This can be explained by preferential neutralization of the C-terminal lysine residue in the depsipeptide, and only partial neutralization in the allamide peptide. Indeed, the most basic residues in these peptides are the two terminal lysines. In the all-amide peptide, the N-terminal Lys should have enhanced basicity due to a favorable solvation of its protonated $\varepsilon$-amine on the $\mathrm{N}$-terminal amine. In contrast, the C-terminal lysine can only solvate its charge on C-terminus and backbone carbonyls, which are energetically less favorable. Since ECD neutralizes the least basic site in multiply charges ions, as it releases the most recombination energy [49], c-ions in the all-amide peptide should have higher abundance than $\mathrm{z}$-fragments, in agreement with the observations. Amide/ester substitution in the fourth peptide bond from the C-terminus reduces the charge solvation opportunities for the C-terminal lysine and thus further reduces its overall basicity. This should enhance the prevalence of the c-fragments.

\section{CAD Results are Best Explained by Differences in Proton Affinity and Product Stability}

The quite substantial effect in CAD of the amide-to-ester bond substitution can be linked to the reduction in the PA of 


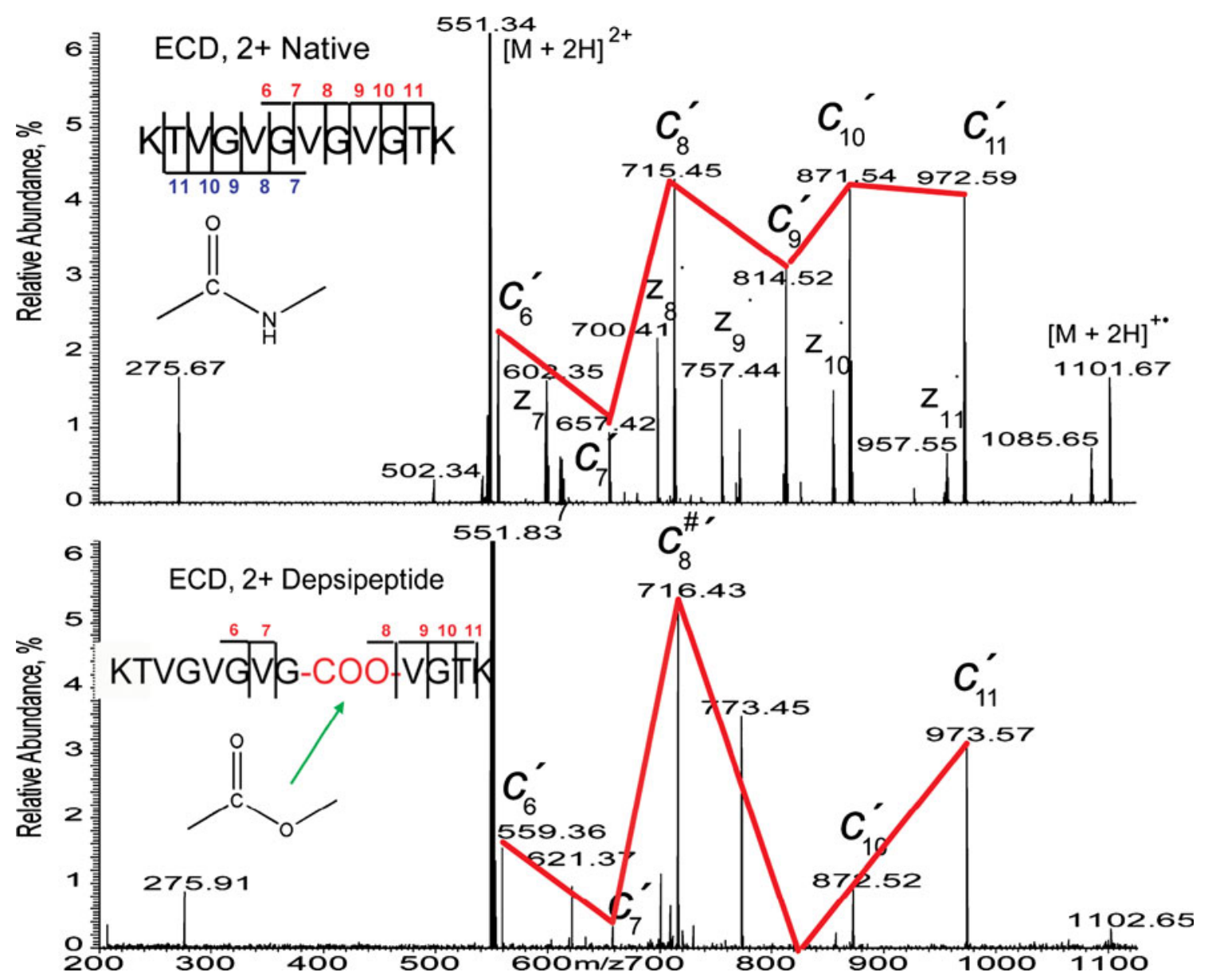

Figure 5. ECD mass spectra of 2+ precursor ions of the semi-tryptic peptide KTVGVGVGVGTK and its depsipeptide analogue KTVGVGVG-COO-VGTK

ester's carbonyl oxygen. Since the likelihood for the peptide bond to be cleaved in CAD is proportional to the basicity of that bond's carbonyl [51], this likelihood should be greatly reduced for esters compared with amides. Moreover, the final step before the peptide bond cleavage in CAD is the protonation of the amide nitrogen; in the ester bond, this step should be the protonation of the oxygen. Comparing the PA values of these two sites using a B3LYP/6-31+G(d,p)//6$311+\mathrm{G}(2 \mathrm{~d}, \mathrm{p})$ level of theory and basis set, we found an energy difference of $98 \mathrm{~kJ} / \mathrm{mol}$ in favor of the amide nitrogen.

From the standpoint of the mobile proton theory [52, 53], the ester carbonyl group appears to present a barrier for further proton migration along the peptide backbone. This is apparent when comparing the product ion distribution of Substance $\mathrm{P}$ and its depsipeptide analogue in charge state 2+ (Figure 3). Here, the cleavage frequency in the depsipeptide drops below that of the all-amide Substance $\mathrm{P}$ for all cleavage sites $\mathrm{C}$-terminal to the ester bond. For the cleavages $\mathrm{N}$-terminal to the ester bond, the frequency is higher than or comparable to that of the all-amide analogue. This result fits well with the reported localization of the two protons in Substance $\mathrm{P} 2+$ ions at $\mathrm{R}^{1}$ and $\mathrm{K}^{3}$ [54]. The proton mobilized from these sites would have to pass the ester bond in order to induce charge-directed cleavages Cterminal to it, which appears to be hampered in the depsipeptide. The impact of the ester on CAD is co-linear with that of the proline residue that is also a barrier for proton transfer, but has a high $\mathrm{PA}$, and as a result the cleavage frequency N-terminal of Pro is enhanced [55].

Another aspect that can influence the cleavage frequency in CAD is the product stability. In the C-terminal fragment, the amide bond cleavage forms an amine group as the Nterminus in an all-amide peptide, while the ester bond cleavage forms an alcohol. The amine has an approximately $150 \mathrm{~kJ} / \mathrm{mol}$ larger PA than the alcohol $\left(\mathrm{CH}_{3} \mathrm{NH}_{2}\right.$ versus $\mathrm{CH}_{3} \mathrm{OH}$ ), which should make charge retention and stabilization more favorable for the amide-produced y ions. Thus, product stability may also play a role in determining the effect of ester/amide bond substitution in CAD.

\section{Conclusions}

In this study, a number of same-sequence peptides and depsipeptides were investigated, comparing ECD with CAD. Changing one backbone bond in a peptide seems to be a 


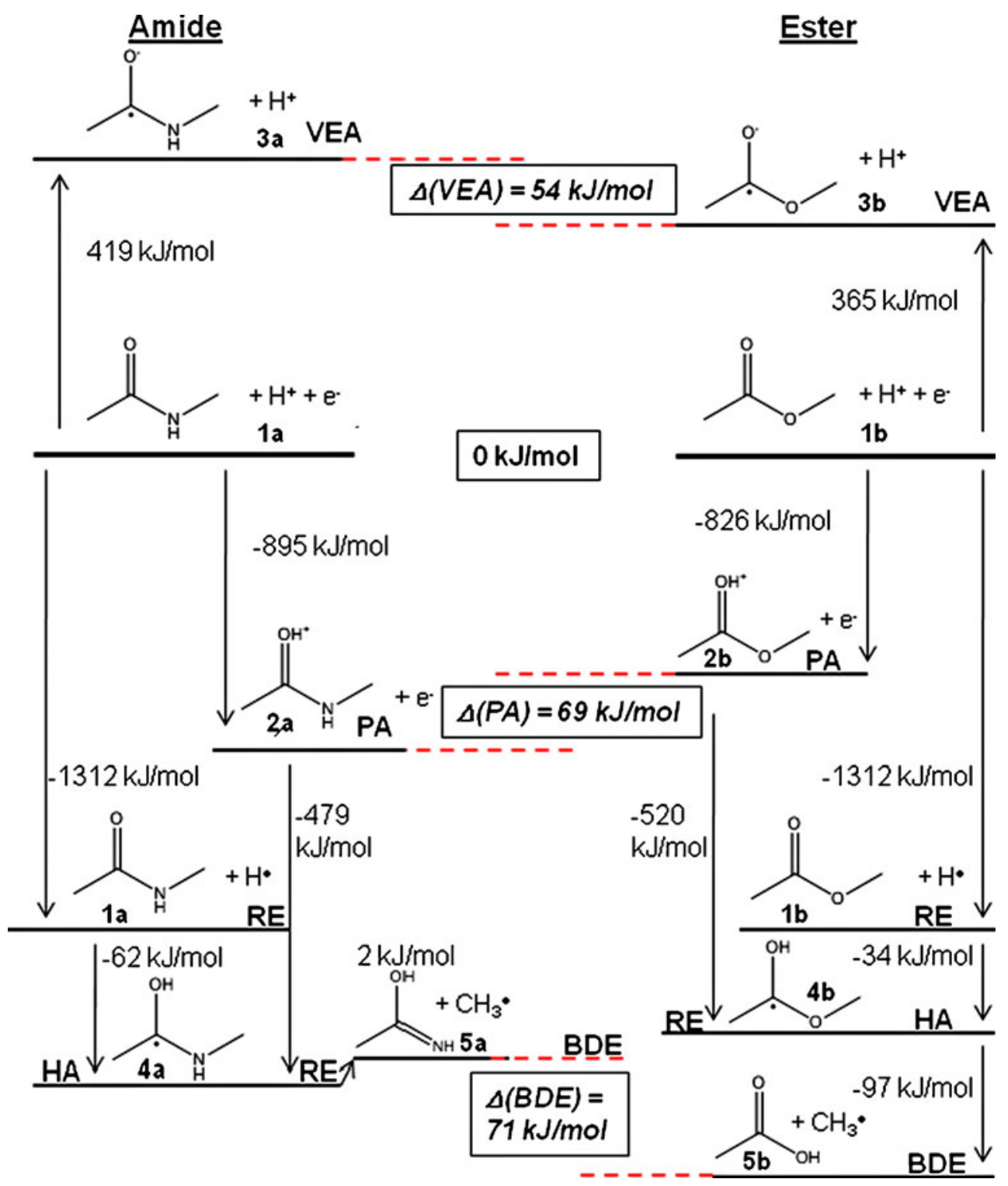

Figure 6. Thermodynamic calculations of the proton affinity (PA), vertical electron affinity (VEA), hydrogen atom affinity (HA), and bond dissociation energy (BDE) for the model system $\mathrm{N}$-methyl acetamide (left) and methyl acetate (right). All values are given in kJ/ mol and are zero-point energy corrected at the B3LYP/6-311++G(2df,2p)//6-31+G(d,p) level of theory except for calculation of the VEA values that were done using MP2/6-31 G(d,p). In the figure, the thermodynamic values are given as: $P A=\Delta H ; V E A=\Delta E ; H A=\Delta E ;$ $\mathrm{BDE}=\Delta \mathrm{E} ; \mathrm{RE}=\Delta \mathrm{H}$

subtle global change. However, both CAD and ECD demonstrated high sensitivity to a single amide-to-ester bond substitution. The general trend in ECD spectra was an enhanced cleavage frequency of the ester bond over that of the amide bond in the same position. In addition, bond substitution reduces the basicity of the nearby protonated residues, which can shift the balance between $\mathrm{N}$ - and $\mathrm{C}$ terminal ECD fragments. An ester bond introduces a "weak link" in the peptide chain in respect to ECD, and thus enhanced $\mathrm{O}-\mathrm{C}_{\alpha}$ bond cleavage reduces the probability of a competing $\mathrm{N}-\mathrm{C}_{\alpha}$ cleavage in neighboring backbone bonds in depsipeptides. The reaction in CAD was opposite to that in ECD, highlighting the complementary nature of the mechanisms of these two techniques. In CAD, the ester bond was cleaved at a minimum level, and it appears to reduce the rate of proton mobilization hampering other backbone cleavages.

Rationalization of these observations was substantiated by thermodynamic calculations. The lower PA of the ester group and higher stability of amide-produced C-terminal fragments that emerged as the main explanations for the reduced cleavage in $\mathrm{CAD}$, while the enhanced stability of the products of ester bond cleavage explains the prevalence of $\mathrm{O}-\mathrm{C}_{\alpha}$ bond cleavage over $\mathrm{N}-\mathrm{C}_{\alpha}$ bond cleavage in ECD. Amide-to-ester backbone bond substitution in peptides presents a novel approach to obtaining novel insights into 
the fundamentally different fragmentation mechanisms of CAD and ECD.

Finally, the enhanced cleavage of $\mathrm{O}-\mathrm{C}_{\alpha}$ bonds increases the potential of electron capture/transfer dissociation for sequencing of naturally occurring depsipeptides.

\section{Acknowledgments}

The authors acknowledge support for this work by the Danish Council for Independent Research, Natural Sciences (FNU grant FK272-08-0044 to FK) and Swedish Research Council (grant 2007-4410 to RZ). They are tremendously thankful to Professor Jack Simons and graduate student Diana Neff at the University of Utah for kindly allowing access to their computer cluster comprising the modified Gaussian version as well as guidance in calculating electron affinities energies. David Good is gratefully acknowledged for careful reading of the manuscript and insightful discussion.

\section{References}

1. Pandey, A., Mann, M.: Proteomics to study genes and genomes. Nature 405(6788), 837-846 (2000)

2. Aebersold, R., Mann, M.: Mass spectrometry-based proteomics. Nature, 422(6928), 198-207 (2003)

3. Zubarev, R.A., Kelleher, N.L., McLafferty, F.W.: Electron capture dissociation of multiply charged protein cations. A nonergodic process. J. Am. Chem. Soc. 120(13), 3265-3266 (1998)

4. Syka, J.E.P., Coon, J.J., Schroeder, M.J., Shabanowitz, J., Hunt, D.F.: Peptide and protein sequence analysis by electron transfer dissociation mass spectrometry. Proc. Natl. Acad. Sci. U.S.A. 101(26), 9528-9533 (2004)

5. Mirgorodskaya, E., Roepstorff, P., Zubarev, R.A.: Localization of Oglycosylation sites in peptides by electron capture dissociation in a Fourier transform mass spectrometer. Anal. Chem. 71(20), 4431-4436 (1999)

6. Stensballe, A., Jensen, O.N., Olsen, J.V., Haselmann, K.F., Zubarev, R. A.: Electron capture dissociation of singly and multiply phosphorylated peptides. Rapid Commun. Mass Spectrom. 14(19), 1793-1800 (2000)

7. Kleinnijenhuis, A.J., Kjeldsen, F., Kalliopolitis, B., Haselmann, K.F., Jensen, O.N.: Anal. Chem. 75(6), 1267-1274 (2007)

8. Kjeldsen, F., Haselmann, K.F., Budnik, B.A., Jensen, F., Zubarev, R. A.: Dissociative capture of hot $(3-13 \mathrm{eV})$ electrons by polypeptide polycations: an efficient process accompanied by secondary fragmentation. Chem. Phys. Lett. 356(3/4), 201-206 (2002)

9. Haddon, W.F., McLafferty, F.W.: J. Am. Chem. Soc. 90, 4745 (1968)

10. Savitski, M.M., Kjeldsen, F., Nielsen, M.L., Zubarev, R.A.: Complementary sequence preferences of electron-capture dissociation and vibrational excitation in fragmentation of polypeptide polycations. Ang. Chem. Int. Ed. 45(32), 5301-5303 (2006)

11. Horn, D.M., Zubarev, R.A., McLafferty, F.W.: Automated de novo sequencing of proteins by tandem high-resolution mass spectrometry. Proc. Natl. Acad. Sci. U.S.A. 97(19), 10313-10317 (2000)

12. Nielsen, M.L., Savitski, M.M., Zubarev, R.A.: Improving protein identification using complementary fragmentation techniques in Fourier transform mass spectrometry. MCP 4(6), 835-845 (2005)

13. Hansen, T.A., Kjeldsen, F., Jensen, O.N.: Generation of Reliable Golden Pairs from Low-resolution Ion Trap Mass Spectrometry Data. 2011, unpublished (Manuscript in preparation)

14. Kruger, N.A., Zubarev, R.A., Horn, D.M., McLafferty, F.W.: Electron capture dissociation of multiply charged peptide cations. Int. J. Mass Spectrom. 187, 787-793 (1999)

15. Syrstad, E.A., Turecek, F.: Hydrogen atom adducts to the amide bond. Generation and energetics of the amino(hydroxy)methyl radical in the gas phase. J. Phys. Chem. A 105(49), 11144-11155 (2001)
16. Syrstad, E.A., Stephens, D.D., Turecek, F.: Hydrogen atom adducts to the amide bond. Generation and energetics of amide radicals in the gas phase. J. Phys. Chem. A 107(1), 115-126 (2003)

17. Neff, D., Smuczynska, S., Simons, J.: Electron shuttling in electron transfer dissociation. Int. J. Mass Spectrom. 283(1/3), 122-134 (2009)

18. Holm, A.I.S., Larsen, M.K., Panja, S., Hvelplund, P., Nielsen, S.B., Leib, R.D., Donald, W.A., Williams, E.R., Hao, C.T., Turecek, F.: Electron capture, femtosecond electron transfer and theory: A study of noncovalent crown ether 1,n-diammonium alkane complexes. Int. J. Mass Spectrom. 276(2/3), 116-126 (2008)

19. Turecek, F., Chung, T.W., Moss, C.L., Wyer, J.A., Ehlerding, A., Holm, A.I.S., Zettergren, H., Nielsen, S.B., Hvelplund, P., ChamotRooke, J., Bythell, B., Paizs, B.: The histidine effect. Electron transfer and capture cause different dissociations and rearrangements of histidine peptide cation-radicals. J. Am. Chem. Soc. 132(31), 1072810740 (2010)

20. Patriksson, A., Adams, C., Kjeldsen, F., Raber, J., van der Spoel, D., Zubarev, R.A.: Prediction of $\mathrm{N}-\mathrm{C}-\longmapsto$ bond cleavage frequencies in electron capture dissociation of Trp-cage dications by force-field molecular dynamics simulations. Int. J. Mass Spectrom. 248(3), 124135 (2006)

21. Kjeldsen, F., Haselmann, K.F., Sorensen, E.S., Zubarev, R.A.: Distinguishing of Ile/Leu amino acid residues in the PP3 protein by (hot) electron capture dissociation in Fourier transform ion cyclotron resonance mass spectrometry. Anal. Chem. 75(6), 1267-1274 (2003)

22. Kjeldsen, F., Zubarev, R.: Secondary losses via $\gamma$-lactam formation in hot electron capture dissociation: A missing link to complete de novo sequencing of proteins? J. Am. Chem. Soc. 125(22), 6628-6629 (2003)

23. Leymarie, N., Costello, C.E., O'Connor, P.B.: Electron capture dissociation initiates a free radical reaction cascade. J. Am. Chem. Soc. 125(29), 8949-8958 (2003)

24. Zubarev, R.A., Kruger, N.A., Fridriksson, E.K., Lewis, M.A., Horn, D. M., Carpenter, B.K., McLafferty, F.W.: Electron capture dissociation of gaseous multiply-charged proteins is favored at disulfide bonds and other sites of high hydrogen atom affinity. J. Am. Chem. Soc. 121(12), 2857-2862 (1999)

25. Demirev, P.A.: Generation of hydrogen radicals for reactivity studies in Fourier transform ion cyclotron resonance mass spectrometry. Rapid Commun. Mass Spectrom. 14(9), 777-781 (2000)

26. Anusiewicz, W., Berdys-Kochanska, J., Simons, J.: Electron attachment step in electron capture dissociation (ECD) and electron transfer dissociation (ETD). J. Phys. Chem. A 109(26), 5801-5813 (2005)

27. Sobczyk, M., Anusiewicz, W., Berdys-Kochanska, J., Sawicka, A., Skurski, P., Simons, J.: Coulomb-assisted dissociative electron attachment: Application to a model peptide. J. Phys. Chem. A 109(1), 250258 (2005)

28. Syrstad, E.A., Turecek, F.: Toward a general mechanism of electron capture dissociation. J. Am. Soc. Mass Spectrom. 16(2), 208-224 (2005)

29. Belyayev, M.A., Cournoyer, J.J., Lin, C., O'Connor, P.B.: The effect of radical trap moieties on electron capture dissociation spectra of Substance P. J. Am. Soc. Mass Spectrom. 17(10), 1428-1436 (2006)

30. Li, X.J., Cournoyer, J.J., Lin, C., O'Connor, P.B.: The effect of fixed charge modifications on electron capture dissociation. J. Am. Soc. Mass Spectrom. 19(10), 1514-1526 (2008)

31. Jones, J.W., Sasaki, T., Goodlett, D.R., Turecek, F.: Electron capture in spin-trap capped peptides. An experimental example of ergodic dissociation in peptide cation-radicals. J. Am. Soc. Mass Spectrom. 18 (3), 432-444 (2007)

32. Sohn, C.H., Chung, C.K., Yin, S., Ramachandran, P., Loo, J.A., Beauchamp, J.L.: Probing the mechanism of electron capture and electron transfer dissociation using tags with variable electron affinity. J. Am. Chem. Soc. 131(15), 5444-5459 (2009)

33. Powers, E.T., Deechongkit, S., Kelly, J.W.: Backbone-backbone Hbonds make context-dependent contributions to protein folding kinetics and thermodynamics: Lessons from amide-to-ester mutations. In Peptide Solvation and H-Bonds 72, 39-78 (2006)

34. Ingwall, R.T., Goodman, M.: Polydepsipeptides. 3. Theoretical conformational-analysis of randomly coiling and ordered depsipeptide chains. Macromolecules 7(5), 598-605 (1974)

35. Kang, Y.K.; Byun, B.J.: Conformational preferences and cis-trans isomerization of L-lactic acid residue. J. Phys. Chem. B 112(30), 91269134 (2008)

36. Cooper, H.J., Hudgins, R.R., Marshall, A.G.: Electron capture dissociation Fourier transform ion cyclotron resonance mass spectrometry of 
cyclodepsipeptides, branched peptides, and $\varepsilon$-peptides. Int. J. Mass Spectrom. 234(1/3), 23-35 (2004)

37. Guan, Z.Q.: Identification and localization of the fatty acid modification in ghrelin by electron capture dissociation. J. Am. Soc. Mass Spectrom. 13(12), 1443-1447 (2002)

38. Thorne, G.C., Ballard, K.D., Gaskell, S.J.: Metastable decomposition of peptide $\mathrm{M}+\mathrm{H}+$ ions via rearrangement involving loss of the C-terminal amino-acid residue. J. Am. Soc. Mass Spectrom. 1(3), 249-257 (1990)

39. Ballard, K.D., Gaskell, S.J.: Intramolecular O-18 isotopic exchange in the gas-phase observed during the tandem mass-spectrometric analysis of peptides. J. Am. Chem. Soc. 114(1), 64-71 (1992)

40. Carpino, L.A., Hann, G.Y.: The 9-fluorenylmethoxycarbonyl aminoprotecting group. J. Org. Chem. 37, 3404-3409 (1972)

41. Frisch, M.J.: Gaussian 03 W, Revision B.05; Gaussian: Pittsburgh, PA, (2003)

42. Boldyrev, A.I., Simons, J.: Isolated So4(2-) and Po4(3-) anions do not exist. J. Phys. Chem. 98(9), 2298-2300 (1994)

43. Whitehead, A., Barrios, R., Simons, J.: Stabilization calculation of the energy and lifetime of metastable SO42-. J. Chem. Phys. 116(7), 2848$2851(2002)$

44. Turecek, F., Yao, C.X.: Hydrogen atom addition to cytosine, 1methylcytosine, and cytosine-water complexes. A computational study of a mechanistic dichotomy. J. Phys. Chem. A 107(43), 9221-9231 (2003)

45. Seydou, M., Modelli, A., Lucas, B., Konate, K., Desfrancois, C., Schermann, J.P.: Electron attachment to strongly polar clusters Formamide molecule and clusters. Eur. Phys. J. D 35(2), 199-205 (2005)

46. Hoffmann, E., Stroobant, V.: Mass Spectrometry Principles and Applications. Second edition (2002)

47. Rienstra-Kiracofe, J.C., Tschumper, G.S., Schaefer, H.F., Nandi, S., Ellison, G.B.: Atomic and molecular electron affinities: Photoelectron experiments and theoretical computations. Chem. Rev. 102(1), 231-282 (2002)

48. Turecek, F.: $\mathrm{N}-\mathrm{C}-\longmapsto$ bond dissociation energies and kinetics in amide and peptide radicals. Is the dissociation a non-ergodic process? $\mathrm{J}$. Am. Chem. Soc. 125(19), 5954-5963 (2003)

49. Kjeldsen, F., Savitski, M.M., Adams, C.M., Zubarev, R.A.: Determination of the location of positive charges in gas-phase polypeptide polycations by tandem mass spectrometry. Int. J. Mass Spectrom. 252 (3), 204-212 (2006)

50. Vorobyev, A., Hamidane, H.B., Tsybin, Y.O.: Electron Capture Dissociation Product Ion Abundances at the $\mathrm{X}$ Amino Acid in RAAAA-X-AAAAK Peptides Correlate with Amino Acid Polarity and Radical Stability. J. Am. Soc. Mass Spectrom. 20(12), 2273-2283 (2009)

51. Savitski, M.M., Kjeldsen, F., Nielsen, M.L., Garbuzynskiy, S.O., Galzitskaya, O.V., Surin, A.K., Zubarev, R.A.: Backbone carbonyl group basicities are related to gas-phase fragmentation of peptides and protein folding. Ang.Chem. Int. Ed. 46(9), 1481-1484 (2007)

52. Dongre, A.R., Jones, J.L., Somogyi, A., Wysocki, V.H.: Influence of peptide composition, gas-phase basicity, and chemical modification on fragmentation efficiency: Evidence for the mobile proton model. $J$. Am. Chem. Soc. 118(35), 8365-8374 (1996)

53. Paizs, B., Suhai, S.: Towards understanding the tandem mass spectra of protonated oligopeptides. 1: Mechanism of amide bond cleavage. J. Am. Soc. Mass Spectrom. 15(1), 103-113 (2004)

54. Gill, A.C., Jennings, K.R., Wyttenbach, T., Bowers, M.T.: Conformations of biopolymers in the gas phase: a new mass spectrometric method. Int. J. Mass Spectrom. 196, 685-697 (2000)

55. Schwartz, B.L., Bursey, M.M.: Some proline substituent effects in the tandem mass-spectrum of protonated pentaalanine. Biol. Mass Spectrom. 21(2), 92-96 (1992) 